\title{
Applications pédagogiques de la séquence filmique en classes romandes : histoire et perspectives
}

\section{Séverine Graff}

\section{(2) OpenEdition}

1 Journals

Édition électronique

URL : https://journals.openedition.org/decadrages/829

DOI : $10.4000 /$ decadrages.829

ISSN : 2297-5977

Éditeur

Association Décadrages

\section{Édition imprimée}

Date de publication : 1 octobre 2015

Pagination : 46-56

ISBN : 9782970096313

ISSN : 2235-7823

\section{Référence électronique}

Séverine Graff, « Applications pédagogiques de la séquence filmique en classes romandes : histoire et perspectives », Décadrages [En ligne], 31 | 2015, mis en ligne le 29 mai 2018, consulté le 25 mars 2023. URL : http://journals.openedition.org/decadrages/829; DOI : https://doi.org/10.4000/decadrages.829

Ce document a été généré automatiquement le 25 mars 2023.

\section{(c) (i) (8)}

Creative Commons - Attribution - Pas d'Utilisation Commerciale - Partage dans les Mêmes Conditions 4.0 International - CC BY-NC-SA 4.0

https://creativecommons.org/licenses/by-nc-sa/4.0/ 


\title{
Applications pédagogiques de la séquence filmique en classes romandes : histoire et perspectives
}

\author{
Séverine Graff
}

1 POUR QUELS OBJECTIFS CONVIENT-IL d'intégrer le cinéma durant le temps scolaire ? Cet article a pour ambition d'esquisser une réponse possible à cette vaste question via la présentation d'un projet du Centre d'études cinématographiques (CEC) de l'Université de Lausanne intitulé Séquences le film au service de l'analyse littéraire. Ce programme met à la disposition des enseignants de français une vingtaine de dossiers pédagogiques liés à des adaptations d'œuvres classiques de la fin du cursus scolaire. Séquences le film au service de l'analyse littéraire comprend trois piliers: les analyses des extraits de films selon une perspective narratologique, stylistique ou thématique destinées aux enseignants; les pistes didactiques et des exercices pour faire travailler les élèves sur les séquences; et enfin les extraits filmiques cités transmis aux enseignants suisses sous la forme de fichiers numériques ${ }^{1}$. Le cinéma, sous forme de brefs extraits, est donc mobilisé pour servir l'analyse littéraire. Cette perspective transversale s'inscrit dans une tradition suisse de l'usage du film en classe. Par un état des lieux fragmentaire ${ }^{2}$, j'aimerais évoquer cette histoire depuis les années1960, esquisser une rapide comparaison avec nos voisins français, puis poser les ambitions didactiques du projet Séquences et le type d'activités proposées aux élèves.

\section{"S'en protéger et en profiter » : du cinéma aux mass- médias}

2 En Suisse romande comme dans de nombreux pays, l'approche éducative du cinéma est traversé par deux tendances: l'éducation à visée cinéphilique qui se donne pour mission de guider les élèves vers de "grandes œuvres », et une éducation aux médias qui consiste à fournir aux jeunes de meilleurs outils pour faire face à ce que l'on nomme, suivant les époques, "mass-médias», «communications de masse» ou 
"nouveaux médias». Cette seconde tendance s'accompagne historiquement d'un discours alarmiste bien antérieur à l'émergence des réseaux sociaux ou même d'internet. En 1968 déjà, lors de l'ouverture à Lausanne du Centre d'initiation au cinéma que nous présenterons ci-après, le journaliste couvrant l'événement s'inquiétait : « Les enfants sont tellement assaillis d'images qu'on peut en effet se demander s'il est juste de faire le jeu de cette avalanche dévastatrice » soulignant dès lors la nécessité de leur " apprendre à regarder et à lire les images. $»^{3}$

En Suisse romande, le début des années1960 est surtout marqué par l'institutionnalisation des structures d'initiation au cinéma, des organismes portés par le modèle des ciné-clubs 4 . Il s'agit d'aider les établissements scolaires à organiser des projections et surtout de permettre aux élèves d'aiguiser leur regard en se fondant sur quelques notions de la "grammaire cinématographique ", supposément introduite en classe par l'enseignant. Notons par exemple la création en 1966 à Fribourg de la Commission d'éducation cinématographique, suivi l'année suivante du Centre vaudois d'initiation au cinéma (CIC) financés par les départements de l'Instruction publique ${ }^{5}$. Ces projets sont marqués par les trois composantes de la tradition des réseaux de cinéclubs : la mise à disposition de films, la création de matériel d'accompagnement et la formation des maîtres, chargés d'enseigner ensuite aux élèves la critique, l'analyse, voire la réalisation. Comme à Fribourg, l'implication des enseignants est l'un des objectifs du Centre d'initiation au cinéma (CIC) de Lausanne que souligne un journaliste chargé de couvrir l'inauguration en 1967 :

« Dans certains pays, il existe des centres très semblables à celui-ci [CIC vaudois] mais qui sont chargés d'initier directement les élèves, le maître ne participant pas à cette initiation. Ici, on veut au contraire permettre aux maîtres d'initier eux-mêmes leur classe au langage cinématographique, pour ne pas accentuer la coupure entre l'école et le cinéma. $»^{6}$

C'est sans doute la spécificité initiale des deux organismes de proposer d'emblée d'introduire le film dans un dispositif scolaire classique (Fribourg consacrera durant quelques années une heure hebdomadaire au Secondaire à l'enseignement du cinéma). Mais ces projets, portés à leurs débuts par une tradition cinéphilique (amener l'élève à voir et à commenter de " grands » films et à s'initier à la réalisation), opèrent dans les années 1970-1980 un changement à la fois d'objet et de perspectives pédagogiques. Le cinéma, entendu comme un long métrage projeté, devient très minoritaire au sein de ce que l'on appelle alors les «mass-médias»: la presse écrite, la publicité, la bande dessinée, la radio et la télévision. Citons en exemple le rapport de 1982 pour la commission intercantonale romande de coordination de l'enseignement intitulé "éducation aux médias ", un document qui envisage le film cinématographique comme une partie minoritaire du médium télévisuel: «La TV englobe le film puisque ce dernier fait partie des programmes [télévisuels] $]^{7}$. Le nom des organismes jusqu'alors dévolus à l'éducation au cinéma change en conséquence : la Commission d'éducation cinématographique fribourgeoise devient en 1975 Centre d'initiation aux mass-médias, son homologue vaudois Centre d'initiation au cinéma se transforme en Centre d'initiation aux communications de masse en 1988. Ces initiatives romandes se regroupent autour d'une double volonté didactique.

5 Premièrement, l'objectif est d'apprendre aux élèves à décoder des messages audiovisuels, autrement dit à éveiller chez eux un esprit critique face à un environnement multimédiatique propre à la société de consommation (beaucoup d'activités analytiques sont alors dévolues à décrypter des messages publicitaires). Ce 
changement d'ambition didactique est parfaitement synthétisé par un journaliste à propos de l'impact des activités du Centre vaudois sur les élèves qui offrent de " perdre la vulnérabilité d'un consommateur d'images succombant à n'importe quelle sollicitation. [...] Il s'agit d'aider les jeunes spectateurs à maîtriser les injonctions de l'image $»^{8}$. Cette peur n'est en réalité pas nouvelle, mais elle infuse particulièrement les discours sur les "mass-médias" de cette époque. L'acronyme de la structure qui coordonne ces centres cantonaux, le GRAVE (Groupe romand de l'audiovisuel à l'école), en témoigne.

6 Deuxièmement, devant l'impossibilité de consacrer un enseignement spécifique à cette question dans tous les cantons, les différents centres initient des projets visant à inclure l'analyse audiovisuelle et la production de projets réalisés par les élèves dans les programmes habituels. Se met alors en place une pédagogie de l'image introduite dans une perspective transversale. Mais quelles activités sont-elles proposées aux classes?

\section{L'exemple des mallettes pédagogique du CIC}

7 Outre la formation des enseignants et le prêt de matériel de réalisation, le Centre vaudois met à la disposition des classes plusieurs mallettes pédagogiques permettant de faire travailler les élèves sur des supports audiovisuels9. Ces mallettes proposent à l'enseignant une séquence didactique complète (souvent une dizaine de leçons). Prenons un exemple: le maître de français souhaitant aborder la narration avec sa classe est guidé grâce au matériel de la mallette pour travailler sur des phénomènes comme la vitesse de narration, le retour en arrière, l'ordre du récit, le suspense. Les activités, organisées selon un plan précis, ne se cantonnent pas à l'analyse d'un texte mais croisent une multitude de supports allant de la bande dessinée, des diapositives aux extraits de films télévisuels ou cinématographiques en VHS. Outre ce matériel multimédia, la mallette comprend aussi des activités précises pour accompagner les élèves dans l'analyse (un chronomètre, des schémas d'analyse pour les séquences, des fiches à remplir). Par ailleurs, la classe est aussi invitée à réaliser des films, soit de manière virtuelle (comme l'esquisse d'un générique, le dessin d'un story-board), soit réellement lors d'une journée passée au studio du Centre où sont effectués le tournage et le montage du court métrage.

8 Le Centre propose deux innovations : la transmission d'activités «clé en main » et l'introduction des médias audiovisuels au service d'autres apprentissages. Son directeur René Schnorf souligne cette double spécificité : «Le centre d'initiation aux médias a dû développer une structure permettant aux enseignants de toutes branches, mais en priorité de français, de mettre en pratique le programme [vaudois d'éducation aux médias]. Ainsi [...] élabore-t-il des cahiers d'activités et des mallettes pédagogiques. $»^{10}$ Une majorité des exercices multimédias est ainsi dévolue à l'enseignement du français. Cette particularité s'explique par l'histoire de la didactique de cette branche qui, depuis le tournant des années1980, délaisse la stricte étude de la grande littérature pour amener les élèves à travailler et à produire des textes aux genres variés. Portées par la double dynamique d'un élargissement des objets étudiés et par le souci du «faire faire » les élèves, les mallettes du CIC rencontreront un écho particulier dans les cours de français dans le canton de Vaud entre 1970 et 2000, bien qu'il soit délicat d'estimer dans quelle mesure l'usage des mallettes est véritablement 
entré dans la pratique. Les années 2000 marquent l'absorbation du cinéma dans les MITIC que nous évoquerons après un bref détour comparatif avec la place du cinéma en France durant les classes de littérature.

\section{L'usage du film en France dans les cours et les manuels de littérature}

Entendu comme une manifestation langagière, le cinéma de fiction occupe en France une place non négligeable dans les programmes officiels de littérature en tant que support d'enseignement, une position que synthétise le chercheur Philippe Bourdier : «Le film représente, parmi d'autres recours pédagogiques, un moyen d'enseigner 〈autrement〉. ${ }^{11}$ Mais quelle place et quelles visées pédagogiques les enseignants de français octroient-ils concrètement au cinéma? En se basant sur une vaste enquête de terrain, le chercheur apporte une réponse plus pessimiste: les films projetés intégralement permettent avant tout d'ouvrir ou de clore l'étude d'un ouvrage. Bourdier regrette que le débat oral croisant les interprétations des élèves ne soit pas une pratique pédagogique plus répandue, afin de diversifier l'usage dominant. Par ailleurs, son étude des manuels scolaires montre que la présence d'images fixes tirées de films est majoritairement cantonnée à la stricte illustration, le choix des films correspondant pour l'essentiel à la ligne promue par le $\mathrm{CNC}$ et ses dispositifs d'éducation à l'image : les " grandes œuvres ». On comprend donc que si le cinéma est avant tout convoqué pour mettre de l'image dans les programmes de français, la démarche pédagogique usuelle ne consiste pas à faire travailler les élèves sur la séquence, mais ponctuellement sur des ersatz de celle-ci. Dans ces manuels en effet, l'élève est au mieux supposé réfléchir à partir des affiches, des captures d'écran et de dialogues retranscrits. Il apprend certes quelques éléments du vocabulaire de description filmique, mais n'est pas invité à analyser la séquence dans sa durée puisque cette dernière n'est ni transmise aux enseignants ni analysée à des fins pédagogiques. Outre les manuels, il faut encore relever les dvd d'extraits dirigés par Alain Bergala pour la collection "Eden cinéma » éditée par le SCÉRÉN-CNDP ${ }^{12}$. Fidèle à son hypothèse $\mathrm{du}$ « choc esthétique » de l'élève face au film, Bergala rejette explicitement le travail sur la séquence, surtout si celui-ci intervient en amont du visionnement : «Il faut que la pensée [de l'élève] vienne de ce qu'on voit et pas du discours sur ce que l'on voit. $»^{13}$ Dans son mouvement d'opposition à l'analyse sémiologique de l'image, Bergala refuse par extension toutes activités analytiques de l'élève sur l'extrait de film, a fortiori à d'autres fins didactiques que de faire des élèves de bons petits cinéphiles (il évoque par exemple la «menace permanente d'instrumentalisation des films qui consiste à les choisir et à les regarder en fonction de la seule exploitation possible de leur sujet, en histoire ou en littérature, par exemple ${ }^{14}$ ). Le cinéma à l'école ? Oui, mais à condition que l'institution se tienne en retrait afin de ne pas entraver la «sidération » supposée intervenir entre l'élève et le « $7^{\mathrm{e}}$ art ».

10 La place du cinéma dans l'enseignement de la littérature en France frappe certes par son importance quantitative dans les manuels et dans les programmes, ce qui dénote la légitimité culturelle d'un certain cinéma, mais interpelle par la faible exploitation didactique des films proposés. 


\section{Suisse : le cinéma dans les MITIC}

11 Dès les années1970-1980 en Suisse, l'initiation au cinéma a laissé place à l'éducation aux médias, soit un glissement d'objet mais aussi d'approche: l'image n'est plus simplement visionnée et commentée pour elle-même dans une perspective de formation artistique et citoyenne; l'élève travaille à sa création ou à son étude afin d'aborder les notions de disciplines scolaires classiques (langues, histoire, etc.). Comme nous l'avons évoqué en introduction du présent numéro, la place du cinéma dans le cursus scolaire romand varie considérablement entre les élèves. Cette disparité s'explique par la tradition cinéphile ou cinéphobe des établissements, mais aussi par le rôle flou du film dans les plans d'études. La place du cinéma depuis l'introduction au début des années 2000 des MITIC est encore plus minoritaire que durant les années1970-1980 puisque les «médias et images » (MI) sont désormais couplés avec les technologies de l'information et de la communication (TIC, anciennement l'informatique). L'introduction des MITIC prolonge ainsi le mouvement esquissé dans les années1970-1980: englober le film cinématographique dans d'autres domaines audiovisuels pour laisser aux initiatives extra- ou parascolaires la projection et le commentaire d'un film en entier. A ce glissement conceptuel s'ajoute un changement de pratique, amorcé dans l'éducation aux médias : les MITIC (donc, même de manière fragmentaire, le cinéma) sont pensés à la fois comme un objet d'étude (enseigner le film), comme un vecteur d'information (enseigner par le film) et comme un outil à pratiquer par les élèves (enseigner la réalisation).

12 Dans un récent article paru sur le blog de L'Hebdo, le chroniqueur Jean-Claude Domenjoz déplorait en conclusion : « La dimension 〈MI〉 (médias et images) peine à être reconnue. La communauté éducative montre peu d'intérêt pour la pédagogie de l'image. Celle-ci nécessite pourtant des connaissances spécifiques et la mise en œuvre de stratégies didactiques appropriées. $»^{15}$ Cette réalité que reconnaissent volontiers les acteurs du terrain s'explique par le fait que les enseignants mobilisés autour du cinéma dans les années1970-1980 partent à la retraite, et les nouvelles générations de praticiens peinent parfois avec les exigences du plan d'études romand (PER) : les MITIC ne sont pas une branche dans la grille horaire, les enseignants de toutes les disciplines sont supposés accompagner les élèves dans la réalisation de projets et l'analyse des médias. Le PER précise par exemple que les élèves doivent apprendre à " décoder la mise en scène de divers type de message", notamment via «l'exploration des principaux éléments qui composent une image fixe ou animée (cadrage, couleur, lumière, profondeur de champ, rythme, mouvement, champ/hors-champ, plans, mise en scène) et du rapport entre l'image et le son ${ }^{16}$. Saluons la présence de ces notions dans le plan d'études qui invite à rendre les élèves conscients des pratiques filmiques, mais relevons tout de même une contradiction : l'image animée serait un objet qu'il est nécessaire d'enseigner. Or, la formation de base en MITIC pour les futurs enseignants romands demeure marginale, voire optionnelle ${ }^{17}$. Quand elle a lieu, cette formation devrait comprendre pour le cinéma (qui ne constitue rappelons-le qu'une petite partie de l'enseignement transversal MITIC) à la fois une introduction à l'analyse de séquences et des outils pour didactiser ces notions. En clair, les enseignants sont supposés enseigner une matière complexe pour laquelle ils ont souvent à peine été formés.

13 Faire travailler une classe sur de l'analyse de séquence est un exercice difficile. Car il ne suffit pas de montrer une profondeur de champ dans Citizen Kane pour que les élèves 
comprennent cette pratique filmique, intègrent ses enjeux en termes de composition de plan et puissent éventuellement reconnaître dans un autre film l'étagement des informations dans le champ et lui donner sens. L'attente qu'il est possible d'avoir à l'égard des enseignants et des élèves semble exagérée si les départements universitaires de cinéma n'accompagnent pas à la fois techniquement, analytiquement et didactiquement l'introduction de la séquence en classe.

\section{Séquences le film au service de l'analyse littéraire}

14 Le Centre d'études cinématographiques (CEC) de l'Université de Lausanne propose différentes activités pour promouvoir la place du cinéma au Secondaire : les formations continues organisées en collaboration avec la HEP Vaud et des ateliers d'analyse de films destinés à compléter ponctuellement les cours de français et d'histoire au gymnase $^{18}$. A ces deux piliers s'ajoute désormais le projet Séquences le film au service de l'analyse littéraire qui transmet directement à l'enseignant de français du matériel (des extraits, des analyses et des activités destinées aux élèves) afin de faire dialoguer les textes littéraires et filmiques. Séquences implique non seulement une transmission succincte du savoir universitaire sous la forme de textes d'accompagnement mais suppose aussi une réélaboration spécifiquement destinée à la classe.

15 Ce projet de transposition est né du constat des défis qu'affrontent les enseignants désireux de varier leur cours de littérature en introduisant des adaptations filmiques : comment faire pour dépasser le «jeu des ressemblances et des différences» entre le roman et son adaptation, la fonction de prolongement illustratif aux lectures menées en amont? Cette attitude cantonne le film à un sous-produit de l'œuvre littéraire. Si une telle préférence se défend dans bien des cas (nous parlons après tout d'un cours de français, non de cinéma), cette hiérarchie entraîne un problème pédagogique : cela empêche un véritable travail de l'élève sur la transposition du littéraire au filmique. Sans analyse formelle, le film n'apporte rien à l'élève du point de vue de sa maîtrise du texte.

16 L'étude individuelle ou en petit groupe d'une adaptation filmique peut aider les élèves en difficulté devant l'analyse littéraire car cet exercice permet de réfléchir au fonctionnement du texte. Dans cette perspective, le projet Séquences sélectionne dix œuvres fréquemment étudiées au Secondaire $\mathrm{II}^{19}$ pour lesquelles ont été rédigées des fiches analytiques pointant par exemple la construction d'un personnage dans différentes adaptations de la même œuvre, le traitement d'un thème littéraire, un procédé narratif ou, plus simplement, la comparaison entre un passage donné et sa transposition cinématographique ou télévisuelle. Toutes ces analyses sont fournies à l'enseignant, accompagnées de pistes didactiques. Car là réside la vraie difficulté : comment faire travailler des élèves sur un flux d'images et de sons, pris dans un défilement continu et montré en projection collective? Ce dispositif classique doit être modifié pour casser la linéarité du film: il s'agit, d'une part, d'exploiter des extraits courts de quelques minutes au maximum, et, d'autre part, de varier la place du cinéma au sein de la séquence didactique littéraire.

17 L'extrait peut précéder la lecture du livre. Cette fonction d'amorce est particulièrement nécessaire pour les lecteurs médiocres qui sont ainsi amenés à faire des prédictions pour se former une image mentale du texte (des habiletés que la chercheuse en didactique Jocelyne Giasson désigne comme «processus 
d'élaboration $»^{20}$ ). Le début du Diable au corps de Claude Autant-Lara (1947) montre par exemple un jeune homme triste traversant à contre-courant une foule en liesse via un long travelling arrière. Il entre dans une maison que vient de quitter un cortège funèbre. Un gros plan du jeune homme est raccordé, via un trucage puis un fondu enchaîné, sur une femme ce qui constitue la première image du flash-back.

18 Le film d'Autant-Lara ne débute pas comme le roman de Radiguet où la structure temporelle est linéaire. On peut donc s'en servir pour amener les élèves à formuler des hypothèses quant à leur lecture future. Expliquer la tristesse du jeune homme, la joie de la foule, les rapports entre les personnages : autant d'hypothèses que l'enseignant se gardera de commenter, laissant la lecture individuelle y répondre progressivement. La même séquence sera à nouveau convoquée plusieurs semaines plus tard en guise de conclusion de l'œuvre pour synthétiser les thèmes du roman. Grâce aux longs mouvements de caméra et aux temporalités narratives, l'incipit du film oppose François pleurant Marthe et la foule grisée par la victoire. L'analyse collective de cet extrait résume le thème du roman : l'amour du couple interdit à contre-courant d'une société mobilisée par la guerre.

19 Mais comment faire travailler les élèves sur une séquence pour qu'ils dégagent euxmêmes les éléments formels leur permettant de dépasser une simple comparaison narrative ? Il est profitable qu'ils puissent manipuler eux-mêmes le film pour rompre sa linéarité. Quelques activités se fondent par exemple sur le principe des cercles de lecture $^{21}$ : on rassemble les élèves en petite formation pour qu'ils s'accordent sur le thème de la séquence (par exemple le contraste entre la peine de François et la joie de la foule). Chaque élève du groupe se voit attribuer une tâche différente : commenter la composition d'un plan, décrire les mouvements de caméra et leur importance en fonction de l'hypothèse collective, analyser le jeu d'acteur ou la musique ou le montage, etc.; puis, ils sont invités à mettre en commun leurs réponses avec leurs camarades chargés de travailler les mêmes éléments formels. Les groupes initiaux sont ensuite reformés pour synthétiser leur analyse en s'appuyant sur des captures d'écran commentées. Le détour par le film peut aussi intervenir comme module interne de la séquence didactique consacrée à l'étude d'une œuvre. "Vous êtes directeur de casting, défendez votre actrice en vous appuyant sur le texte original »; « Vous êtes scénariste. Préparez un découpage cinématographique de ces quelques lignes en soulignant dans le texte ce que vous voulez mettre en scène. Vous accorderez un soin particulier à la focalisation. Puis vous esquissez dans la colonne de droite les plans » : ces consignes, qui font appel à des pratiques sociales de référence, obligent les élèves à mobiliser leur aptitude en analyse de texte. Cette exploitation directe donne sens à une activité scolaire dont ils peinent parfois à situer l'utilité.

20 Outre les avantages strictement didactiques, introduire le film en classe permet aussi une valorisation des compétences extra-scolaires des élèves en tant que spectateurs. Les films cités dans les fiches ne sont pas tous des "grandes œuvres". Même des adaptations faibles d'un point de vue cinématographique peuvent déboucher sur des activités productives car même de «mauvaises adaptations » posent d'intéressantes questions dans la transposition des procédés stylistiques.

21 L'exploitation d'extraits de films peut contribuer à une remédiation pédagogique des difficultés d'apprentissage face à l'analyse de texte. Le projet se fonde sur la polysémie du mot séquence en militant pour une introduction régulière de la séquence filmique au sein de la séquence didactique. Fort d'une tradition transversale du cinéma et d'une 
tradition pédagogique romande centrée sur l'activité des élèves, ce projet pense l'extrait et le travail sur celui-ci comme un des outils de connaissance au service de l'enseignement.

\section{NOTES}

1. Les analyses rédigées destinées aux enseignants de français et les activités pour les élèves sont disponibles en ligne sur : www.unil.ch/cec à partir de décembre 2015. 2016 verra l'introduction du second volet du projet : Séquences le film au service de l'analyse historique. Les séquences citées dans ces travaux sont à la disposition des enseignants sur demande auprès de l'auteure du présent article. D'un point de vue légal, ce travail se réfère au droit de citation, article 25 de la loi fédérale sur le droit d'auteur et les droits voisins (art. 25 LDA où l'alinéa1 stipule : « Les citations tirées d'œuvres divulguées sont licites dans la mesure où elles servent de commentaire, de référence ou de démonstration et pour autant que leur emploi en justifie l'étendue ») du moment où l'auteur et la source sont expressément mentionnés. Naturellement, Séquences le film au service de l'analyse littéraire est exclusivement prévu pour un usage pédagogique.

2. Une étude historique exhaustive de l'enseignement du cinéma en Suisse romande reste à faire. Cet article, pensé comme un état des lieux fragmentaire, ne prétend prendre en charge cette tâche qui serait ambitieuse compte tenu des nombreuses initiatives d'introduction du film en classe qui jalonnent l'histoire de l'école romande.

3. A. Mr, « Le CIC a ouvert ses portes », Tribune de Lausanne, 17 février 1968.

4. Ces différents projets romands connaissent de fortes disparités cantonales. Pour un panorama général, je renvoie au Rapport de la commission romande et tessinoise chargée d'étudier les activités cantonales et romandes en matière d'audiovisuel dans l'enseignement, Lausanne, mars 1980 ; archive disponible sur https://edu.ge.ch/site/archiprod/archives-grave/ (consulté le 10août 2015).

5. Pour une étude historique des activités fribourgeoises en matière d'éducation au cinéma, je renvoie à Laurent Diserens, L'Education au cinéma et aux mass-médias dans les écoles du canton de Fribourg. Deux tentatives pour former les élèves à la lecture de l'image, mémoire de licence, Faculté des Lettres, Fribourg, 2003-2004.

6. A.Mr, « Le CIC a ouvert ses portes », op. cit.

7. L'Education aux mass-médias dans la perspective des programmes-cadres du CIRCE III, Neuchâtel, GRAVE, p. 3, février 1983, disponible en ligne sur: wwwedu.ge.ch/sem/production/grave/ archives/GRAVE/agrcirce.pdf.

8. A.Mr, « Le CIC a ouvert ses portes », op.cit.

9. Ces mallettes pédagogiques sont destinées à des élèves de tous niveaux. Le projet Séquences du CEC, lui, s'adresse prioritairement aux étudiants du Secondaire II (15 à 18ans).

10. René Schnorf est le second directeur du Centre depuis 1988. Il succède ainsi à Jean-Pierre Golay.

11. Les programmes officiels demandent d'accorder une importance au film dans les cours de littérature, une position dont la majorité des manuels se font l'écho. Sur les 12manuels de français Secondaire II étudiés par Bourdier, 11consacrent une page sur 20 au cinéma. Voir Philippe Bourdier, Un grand écran pour les Lettres: le cinéma et l'enseignement du français, Paris, L'Harmattan, 2012. 
12. Mehdi Derfoufi présente L'Hypothèse cinéma d'Alain Bergala et y synthétise les conceptions cinéphiliques de l'auteur en matière d'éducation au cinéma dans le présent dossier. J’y renvoie le lecteur pour plus de détails.

13. Alain Bergala cité par Christian Georges dans « Toute pédagogie a pour devoir de ralentir, les images, le temps... ", L'Educateur, octobre 2013, p. 7.

14. Alain Bergala, L'Hypothèse cinéma. Petit traité de transmission du cinéma à l'école et ailleurs, Paris, Cahiers du cinéma, 2006 [2002], p. 40.

15. Jean-Claude Domenjoz, "MITIC : Pour une pédagogie de l'image en Suisse romande ", mis en ligne le 23 août 2015, www.hebdo.ch (consulté le 10 septembre 2015).

16. www.plandetudes.ch/web/guest/FG_21/, rubrique «Education aux médias ", consulté le 11octobre 2015.

17. Deux cours optionnels à 6 crédits sont proposés aux futurs enseignants en Secondaire I et II vaudois à la HEP Vaud. Le CERF de Fribourg exige des étudiants un cours à 2crédits pour les futurs enseignants du Secondaire II ; 3 crédits pour les futurs enseignants du Secondaire I. L'Institut universitaire de formation des enseignants exige le suivi d'un cours à 4 crédits.

18. Pour plus de détails sur cette offre, voir Alain Boillat, Séverine Graff, «L'étude du cinéma, de l'Université aux classes du Secondaire ", Jeunes \& Médias. Les cahiers francophones de l'éducation aux médias, $\mathrm{n}^{\circ}$ 7, 2015.

19. La Princesse de Clèves de Madame de la Fayette, Bel Ami et Boule de Suif de Guy de Maupassant, Madame Bovary de Gustave Flaubert, Cyrano de Bergerac d'Edmond Rostand, La Bête Humaine et Nana d'Emile Zola, La Religieuse de Denis Diderot, Le Diable au corps de Raymond Radiguet et Les Liaisons dangereuses de Pierre Choderlos de Laclos.

20. Jocelyne Giasson, La Compréhension en lecture, Bruxelles, De Bœck, 1990.

21. Un cercle de lecture est un dispositif didactique qui rassemble les élèves en petits groupes hétérogènes afin de leur permettre de construire ensemble des connaissances à partir de textes étudiés. Voir Annette Lafontaine, Serge Terwagne, Sabine Vanhulle, Les Cercles de lecture. Interagir pour développer ensemble des compétences de lecteur, Paris, De Bœck, 2006. 